\title{
Automedicación con AINE en futbolistas profesionales de los equipos de primera división del DMQ
}

\author{
Self-medication with nsaids in professional soccer players of the first division teams of \\ the DMQ
}

\section{Automedicação com nsaids em jogadores profissionais de futebol das equipes da primeira divisão do DMQ}

\author{
María Belén Ríos Quiñónez \\ mblenrios@outlook.com \\ ORCID: 0000-0002-3742-4865
}

Instituto Tecnológico Universitario Cordillera, Ecuador

Recibido 21 de octubre 2020 | Arbitrado y aceptado 19 de diciembre 2020 | Publicado 22 de diciembre 2020

\begin{abstract}
RESUMEN
Introducción: Varios estudios a cargo de la Federación Internacional de Fútbol han identificado el abuso de AINE (Analgésicos Antinflamatorios No Esteroideo) en los futbolistas indicando que casi el $40 \%$ de los jugadores del mundial de 2010 tomaron medicamentos para el dolor antes de cada partido. Objetivo: identificar el nivel de conocimiento sobre AINE que presentan los futbolistas profesionales de las plantillas de primera división, a través del análisis y realidad de cada equipo. Método: se aplicaron encuestas validadas por los departamentos médicos de cada club y a través de preguntas abiertas, cerradas y formato de opción múltiple, se determinó que: la edad de los futbolistas profesionales en estudio oscila entre los 15 y 34 años. Resultados: Respecto al conocimiento del grupo farmacológico que contrarresta síntomas de dolor, fiebre e inflamación; se determinó que existe gran confusión e ignorancia en relación a la identificación de medicamentos y su conceptualización. Así mismo, respecto a principios activos que tratan dolor, fiebre e inflamación, se notó preferencia por lbuprofeno, Paracetamol y Diclofenaco. Y que menos del $50 \%$ del total de futbolistas reconocen los efectos adversos de los fármacos en estudio. Conclusión: existen falencias respecto al conocimiento de medicamentos (AINE), y que debido a la premura con la que requieren volver a las canchas, éstos profesionales del deporte, suelen confiar en personas que no se encuentran capacitados en medicina o en temas farmacológicos.
\end{abstract}

Palabras clave: Antiinflamatorios no Esteroideos; Analgésico; Automedicación; Mialgia; Fútbol
MR: Tecnóloga en Administración de Boticas y Farmacias, Máster Internacional en Dirección y Gestión de la Oficina de Farmacia (Esneca), 5 años de experiencia docente. Cursos: Claves para la Innovación en la docencia universitaria, Diplomado en Docencia Universitaria, Revisión de Doćculos científicos Publicación: articulos Publicación Importancia de la profesionalización en la industria farmacéutica: diagnóstico de perfiles laborales del sector farmacéutico en Quito-Ecuador. Instituto Tecnológico Universitario Cordillera. Ecuador.

\begin{abstract}
Introduction: Several studies conducted by the International Football Federation have identified the abuse of NSAIDs (Non-Steroidal Anti-Inflammatory Analgesics) in soccer players, indicating that almost $40 \%$ of the 2010 World Cup players took pain medication before each match. Objective: to identify the level of knowledge about NSAIDs presented by professional footballers of the first division squads, through the analysis and reality of each team. Method: surveys validated by the medical departments of each club were applied and through open and closed questions and multiple choice format, it was determined that: the age of the professional soccer players in the study ranges between 15 and 34 years. Results: Regarding the knowledge of the pharmacological group that counteracts symptoms of pain, fever and inflammation; It was determined that there is great confusion and ignorance in relation to the identification of drugs and their conceptualization. Likewise, with respect to active principles that treat pain, fever and inflammation, a preference was noted for lbuprofen, Paracetamol and Diclofenac. And that less than $50 \%$ of all footballers recognize the adverse effects of the drugs under study. Conclusion: there are shortcomings regarding the knowledge of medications (NSAIDs), and that due to the haste with which they need to return to the courts, these sports professionals often trust people who are not trained in medicine or pharmacological issues.
\end{abstract}

Key words: Non-Steroidal Anti-inflammatory Drugs; Analgesic; Self-medication; Myalgia; Football 
MR: Tecnóloga en Administración de Boticas y Farmacias, Máster Internacional en Dirección y Gestión de la Oficina de Farmacia (Esneca), 5 años de experiencia docente. Cursos: Claves para la Innovación en la docencia universitaria, Diplomado en Docencia Universitaria, Revisión de Doćncios cientícos articulos cientifcos. Publi Importancia de la profesionalización en la industria farmacéutica: diagnóstico de perfiles laborales del sector farmacéutico en Quito-Ecuador. Instituto Tecnológico Universitario Cordillera. Ecuador.

\section{RESUMO}

Introdução: Vários estudos realizados pela Federação Internacional de Futebol identificaram o uso abusivo de NSAIDs (Analgésicos Antiinflamatórios Não Esteróides) em jogadores de futebol, indicando que quase $40 \%$ dos jogadores da Copa do Mundo de 2010 tomavam analgésicos antes de cada partida. Objetivo: identificar o nível de conhecimento sobre os AINE apresentado por futebolistas profissionais das equipes da primeira divisão, por meio da análise e da realidade de cada equipe. Método: foram aplicadas pesquisas validadas pelos departamentos médicos de cada clube e por meio de questões abertas e fechadas e no formato de múltipla escolha, determinou-se que: a idade dos jogadores profissionais de futebol em estudo varia entre 15 e 34 anos. Resultados: Quanto ao conhecimento do grupo farmacológico que neutraliza os sintomas de dor, febre e inflamação; Constatou-se que existe grande confusão e desconhecimento em relação à identificação dos medicamentos e sua conceituação. Da mesma forma, no que diz respeito aos princípios ativos que tratam a dor, febre e inflamação, foi observada uma preferência para ibuprofeno, paracetamol e diclofenaco. E que menos de $50 \%$ de todos os jogadores de futebol reconhecem os efeitos adversos das drogas em estudo. Conclusão: há lacunas no conhecimento sobre medicamentos (NSAIDs) e que, devido à pressa com que precisam retornar às quadras, esses profissionais do esporte costumam confiar em pessoas sem formação em medicina ou farmacologia.

Palavras-chave: Medicamentos antiinflamatórios não esteroidais; Analgésico; Automedicação; Mialgia; Futebol

\section{INTRODUCCIÓN}

$\mathrm{E}$ I fútbol, rey de los deportes, no solo se juega dentro de las canchas; sino también fuera de ellas. A nivel mundial, este deporte es considerado por la gran mayoría de la población como el más practicado y con más aficionados, en relación a otras prácticas de la misma línea competitiva. En el planeta aproximadamente 270 millones de personas están activamente involucradas en este deporte; de éstas, 265 millones juegan al fútbol regularmente de manera profesional, semi-profesional o amateur, considerando tanto a hombres, mujeres, jóvenes y niños (1).

Resulta importante mencionar que visto desde la televisión o en la tribuna de un estadio, el fútbol genera pasiones entre sus miles de aficionados alrededor del mundo; no en vano es uno de los deportes más integrales desarrollado hace más de 140 años en Inglaterra. Dentro de este enorme mundo deportivo, el fútbol mueve gigantescas cantidades de dinero no sólo en contrataciones de jugadores, sino también en auspicios y alianzas comerciales.

Tal como lo menciona el portal web EA SPORTS (2) el mundo del fútbol tiende a ser desconcertante muchas de las veces; puesto que no sólo trata temas netamente deportivos; sino que, puede llegar a indagar en temas médicos - científicos que afectan a la vida de los "elit deportist" en cada equipo a lo largo del mundo.

Tomando esta acotación como antecedente, es importante relacionar lo que detalla la Federación Internacional de Fútbol (3); donde, tras un estudio exhaustivo se anunció el abuso de AINE (Analgésicos Aintiinflamatorios No Esteroideo) en los futbolistas diciendo que: "la carrera de los futbolistas podría estar en peligro por el uso abusivo de analgésicos", dato advertido por Jiri Dvorak, director médico de la FIFA, quien encontró además, que casi el $40 \%$ de los jugadores del Mundial de 2010 tomaron medicamentos para el dolor antes de cada partido, según revelaron los resultados publicados en la revista British Journal of 
Sports Medicine y de los que se hace eco la BBC de Londres.

Siguiendo la misma línea investigativa, los médicos del organismo que rige el fútbol (FIFA) publicaron este año un estudio sobre las sustancias tomadas por los jugadores en los dos últimos mundiales. La conclusión es clara. "La medicación en el fútbol es excesiva y alarmante; dudamos que el uso sea sólo terapéutico", afirma. El informe acoge 2.944 muestras entregadas por los médicos de cada selección, y apunta al uso desmesurado de antiinflamatorios no esteroideos, tipo Voltarén. Además, se tienen los siguientes datos: más del $50 \%$ de los jugadores los tomaron en ambos mundiales, y el 30,8\% lo hizo antes de cada partido. Más preocupante aún, es que la FIFA apunta a una selección, en la que 22 de sus 23 jugadores los usaban sistemáticamente antes de cada encuentro (4).

Por otro lado, es relevante exponer lo que detalla Medic Sports (5), donde se menciona que: "Es incuestionable que este tipo de aintiinflamatorios no Esteroideo alivian el dolor, pero su capacidad de cura es dudosa y un uso prolongado afecta al riñón", advierte la FIFA. "El deporte también debe significar salud (...)".

Una vez descrita la cuestión mundial, es importante analizar lo que sucede a nivel de Sudamérica, donde el fútbol se vive de una manera distinta y relativamente más apasionada que en cualquier otra parte del planeta; el hecho de mantener tanta competitividad entre los distintos países hace que sus respectivos equipos se preparen de manera constante y sobre todo exigente, para así enfrentar las distintas competencias que presenta el continente.

Es así, que en el Ecuador existen 12 equipos que conforman la seria $\mathrm{A}$ del Campeonato Nacional, de los cuales 5 pertenecen al Distrito Metropolitano de Quito (6). Resulta importante recalcar que en nuestro país el fanatismo por cada partido de fútbol se vive a flor de piel, siendo un medio de entretenimiento para chicos y grandes cada semana.

Específicamente en Quito, se concentra una gran "pasión futbolera", hecho que se observa antes, durante y después de cada partido; no solamente en los estadios; sino fuera de ellos, convirtiendo a las hinchadas en entes estratégicos para el aliento de cada partido, quienes hacen uso de redes sociales (Twitter, Facebook, Instagram) o se acercan a los campos de entrenamiento para generar "presión" a los futbolistas y que así éstos se comprometan a ganar o ganar cada encuentro como si se tratase de una final.

Por ende, toda esta gama de particularidades obliga a que los responsables de dar alegrías a los equipos e hinchadas: los futbolistas, conlleven grandes presiones por lograr la victoria de sus clubes.

En relación a lo señalado, la información expuesta en investigaciones de la FIFA puede ligarse con la realidad de los clubes ecuatorianos, donde se reitera que la presión que poseen los futbolistas por obtener resultados positivos en los encuentros y sobre todo por poseer un rendimiento físico excelente y no lesionarse, los obliga a consumir medicamentos para acelerar su recuperación o tan solo para lograr ser inmunes al dolor; se trata de la administración desmedida de los AINE (analgésicos antiinflamatorios no Esteroideo).

Es así, que surgen varias interrogantes asociadas a cuánto saben los futbolistas de las plantillas principales de los equipos de primera división del Distrito Metropolitano de Quito - Zona 9: Club Deportivo de la Universidad Católica (UC), Club Deportivo El Nacional (EN), Liga Deportiva Universitaria de Quito (LDUQ) e Independiente del Valle (IV) acerca de este tipo de medicamentos: usos, vías de administración, efectos 
secundarios, formas farmacéuticas e ideas generales asociadas a la auto medicación.

Para dar respuesta a la problemática mediante el objetivo se identifica el nivel de conocimiento sobre AINE que presentan los futbolistas profesionales de las plantillas de primera división, a través del análisis y realidad de cada equipo $y$, reconocer los diferentes tipos de AINE utilizados con mayor frecuencia por los futbolistas, vías de administración y las afecciones que pueden provocar en el organismo.

Tomando en cuenta que la presión y nivel de exigencia en el campo futbolístico son más evidentes en la actualidad; y que la fuerte competencia que se presenta a diario dentro y fuera de la cancha obliga a los futbolistas a tomar decisiones farmacéuticas no tan profesionales; mismas que reflejarán repercusiones a largo plazo para su salud por el hecho de realizar entrenamientos a diario, tan complejos y potentes que los vuelve propensos a lesionarse en partidos oficiales; se consideró necesario realizar una investigación exhaustiva asociada al análisis de conocimiento de AINE y nivel de uso de los mismos; ya que sin duda el hecho de que estos medicamentos sean de libre venta $u$ OTC y puedan encontrárselos en distintos establecimientos farmacéuticos (autorizados o no para la comercialización de los mismos); otorgan un fácil acceso a ellos; provocando una costumbre de auto medicación sin fundamento médico alguno.

En definitiva, el análisis de los datos encontrados, serán de gran aporte para las áreas médicas y farmacéuticas asociadas al deporte en general; pues no suele ser tan fácil discernir entre la vida personal y profesional de aquellos deportistas que dedican su vida a estas labores. Estos temas no suelen estar en la palestra de los temas farmacéuticos; pero es importante conocer el nivel de educación en temas sanitarios de estos grupos sociales para mejorar sus costumbres farmacéuticas y sobre todo para mejorar su calidad de vida.

\section{MATERIALES Y MÉTODOS}

$\mathrm{L}$ a investigación de fue de tipo documental, descriptiva; pues en primera instancia se analizó la información escrita sobre el tema objeto de estudio imprescindible para estudiar los fenómenos. Para la recopilación de datos se aplicaron encuestas validadas por los departamentos médicos de cada club y a través de un cuestionario con preguntas abiertas, cerradas y formato de opción múltiple que incluyeron: edad de los encuestados, identificación y frecuencia de uso de fármacos que tratan fiebre-dolor e inflamación, efectos adversos, profesionales a los que acuden ante una dolencia, vías de administración y percepción sobre la automedicación, se exploró el nivel de conocimiento que tienen los futbolistas acerca de los AINE y el uso que le dan a los mismos.

En lo referente a la población de estudio; se trabajó con la totalidad de misma, debido a que fue reducida a 30 jugadores por cada equipo: Club Deportivo de la Universidad Católica (UC), Club Deportivo El Nacional (EN), Liga Deportiva Universitaria de Quito (LDUQ) e Independiente del Valle (IV); de donde se obtuvo datos certeros y reales para el sustento de la investigación.

\section{RESULTADOS Y DISCUSIÓN}

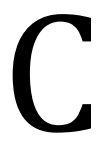
omo dato primordial, se consultó las edades de los futbolistas de los Clubes Deportivos de la Universidad Católica (UC), El Nacional (EN), Liga Deportiva Universitaria de Quito (LDUQ) e Independiente del Valle (IV) de estas 
plantillas principales; fue de gran importancia para saber desde cuándo se accedía a este tipo de medicamentos. Para ello, el detalle en la Tabla 1.

Tabla 1. Edades de los jugadores pertenecientes a plantillas principales.

\begin{tabular}{|c|c|c|c|c|c|c|c|c|}
\hline \multirow{2}{*}{ Edad } & \multicolumn{2}{|l|}{ UC } & \multicolumn{2}{|l|}{ EN } & \multicolumn{2}{|c|}{ LDUQ } & \multicolumn{2}{|l|}{ IV } \\
\hline & Frecuencia & $\%$ & Frecuencia & $\%$ & Frecuencia & $\%$ & Frecuencia & $\%$ \\
\hline 15 & - & - & 1 & 3 & - & - & - & - \\
\hline 16 & - & - & 3 & 10 & - & - & - & - \\
\hline 17 & 1 & 3 & 1 & 3 & - & - & - & - \\
\hline 18 & 1 & 3 & 5 & 17 & 7 & 23 & 2 & 7 \\
\hline 19 & 4 & 13 & 2 & 7 & 9 & 30 & - & - \\
\hline 20 & - & - & 4 & 13 & 3 & 10 & - & - \\
\hline 21 & 1 & 3 & 6 & 20 & - & - & 2 & 7 \\
\hline 22 & 2 & 7 & 1 & 3 & 1 & 3 & - & - \\
\hline 23 & 1 & 3 & 2 & 7 & - & - & 7 & 23 \\
\hline 24 & 1 & 3 & 1 & 3 & 3 & 10 & 1 & 3 \\
\hline 25 & 5 & 17 & - & - & - & - & 2 & 7 \\
\hline 26 & 2 & 7 & 1 & 3 & 3 & 10 & 3 & 10 \\
\hline 27 & 1 & 3 & - & - & 1 & 3 & 6 & 20 \\
\hline 28 & 2 & 7 & 1 & 3 & 2 & 7 & 2 & 7 \\
\hline 29 & - & - & - & - & - & - & 3 & 10 \\
\hline 30 & 2 & 7 & - & - & - & - & 1 & 3 \\
\hline 31 & 2 & 7 & - & - & - & - & - & - \\
\hline 32 & 2 & 7 & 2 & 7 & - & - & - & - \\
\hline 33 & 2 & 7 & - & - & 1 & 3 & 1 & 3 \\
\hline 34 & 1 & 3 & - & - & - & - & - & - \\
\hline Total & 30 & $100 \%$ & 30 & $100 \%$ & 30 & $100 \%$ & 30 & $100 \%$ \\
\hline
\end{tabular}

Haciendo referencia a la encuesta orientada a solicitar información respecto a qué tipo de medicamentos son los que alivian dolor, fiebre e inflamación; se obtuvieron los siguientes resultados, en el Club Deportivo Universidad Católica un 87\% (26 personas) afirman que son los antibióticos los fármacos que alivian dolor, fiebre e inflamación. En el caso del Club Deportivo El Nacional, el $90 \% \quad(27$ individuos) de encuestados consideran que son los antibióticos los fármacos que tratan esas dolencias. En Liga Deportiva Universitaria de Quito, 20 futbolistas representados con un $66 \%$ aducen que son los antibióticos aquellos medicamentos que tratan dolor, fiebre e inflamación. Finalmente, en Independiente del Valle, el $30 \%$ (19 jugadores) concuerdan que los AINE son los fármacos indicados para tratar los síntomas descritos en la pregunta.

En lo que concierne a la selección de medicamentos con los que tratarían dolor, fiebre e inflamación (pregunta 3); se exponen los siguientes resultados: (Tabla 2). 
Tabla 2. Identificación de fármacos que tratarían dolor, fiebre e inflamación.

\begin{tabular}{|c|c|c|c|c|c|c|c|c|}
\hline \multirow{2}{*}{ Opciones } & \multicolumn{2}{|l|}{ UC } & \multicolumn{2}{|c|}{ EN } & \multicolumn{2}{|c|}{ LDUQ } & \multicolumn{2}{|l|}{ IV } \\
\hline & Frecuencia & $\%$ & Frecuencia & $\%$ & Frecuencia & $\%$ & Frecuencia & $\%$ \\
\hline Ibuprofeno & 15 & 21 & 18 & 26 & 15 & 29 & 7 & 14 \\
\hline Paracetamol & 19 & 43 & 17 & 25 & 19 & 37 & 8 & 16 \\
\hline Diclofenaco & 6 & 20 & 14 & 20 & 6 & 12 & 21 & 43 \\
\hline Meloxicam & 3 & 10 & 14 & 20 & 3 & 6 & 12 & 25 \\
\hline Tenoxicam & 0 & 0 & 1 & 1 & 0 & 0 & 1 & 2 \\
\hline Aspirina & 8 & 6 & 5 & 7 & 8 & 16 & 0 & 0 \\
\hline Total & 51 & $100 \%$ & 69 & $100 \%$ & 50 & $100 \%$ & 49 & $100 \%$ \\
\hline
\end{tabular}

Otra interrogante planteada fue la frecuencia de uso de: Ibuprofeno, Paracetamol, Diclofenaco, Meloxicam, Tenoxicam y Aspirina. Los resultados se detallan así: de manera coincidente, en Universidad Católica y Club Deportivo El Nacional con un $80 \%$ (24 jugadores), Liga Deportiva Universitaria con el 90\% (27 futbolistas) e Independiente del Valle con un $87 \%$ (26 jugadores), indican que los consumen nada más cuando se presenta una lesión, pues dejan todo en la cancha o los entrenamientos son muy pesados; por lo demás no los consideran necesarios.

Por otro lado, la Tabla 3 refleja los resultados obtenidos tras verificar a qué establecimiento o profesional sanitario acude cada futbolista cuando presenta alguna dolencia y requiere de fármacos que alivien la misma. 
Tabla 3. Identificación de profesional sanitario o establecimiento al que acuden los futbolistas.

\begin{tabular}{|c|c|c|c|c|c|c|c|c|}
\hline \multirow{2}{*}{ Opciones } & \multicolumn{2}{|l|}{ UC } & \multicolumn{2}{|l|}{ EN } & \multicolumn{2}{|l|}{ LDUQ } & \multicolumn{2}{|l|}{ IV } \\
\hline & Frecuencia & $\%$ & Frecuencia & $\%$ & Frecuencia & $\%$ & Frecuencia & $\%$ \\
\hline $\begin{array}{l}\text { Toma } \\
\text { únicamente los } \\
\text { medicamentos } \\
\text { que le indica el } \\
\text { doctor del Club }\end{array}$ & 30 & 100 & 26 & 87 & 27 & 90 & 28 & 93 \\
\hline $\begin{array}{c}\text { Acude a la } \\
\text { farmacia y } \\
\text { acepta la } \\
\text { sugerencia de } \\
\text { los } \\
\text { colaboradores } \\
\text { del } \\
\text { establecimiento }\end{array}$ & 0 & 0 & 4 & 13 & 3 & 10 & 2 & 7 \\
\hline Total & 30 & $100 \%$ & 30 & $100 \%$ & & & & \\
\hline
\end{tabular}

En lo referente a vías de mencionan que suelen tomar los administración de fármacos que alivian medicamentos por vía oral. Pero es dolor, fiebre e inflamación, planteada en importante destacar que, en porcentajes otra pregunta; los resultados son los siguientes: Universidad Católica con el $82 \%$ (27 jugadores), Independiente del Valle con un $77 \%$ (24 futbolistas), Club Deportivo El Nacional con $70 \% \quad(26$ jugadores) y Liga Deportiva Universitaria de Quito con el 91\% (29 futbolistas); variados, la vía intramuscular es la segunda opción en todos los equipos.

Para analizar las respuestas respecto a efectos adversos que causan los medicamentos que alivian dolor, fiebre e inflamación (AINE), se presenta la Tabla 4. 
Tabla 4. Percepción sobre efectos adversos de los AINE.

\begin{tabular}{|c|c|c|c|c|c|c|c|c|}
\hline \multirow{2}{*}{ Opciones } & \multicolumn{2}{|l|}{ UC } & \multicolumn{2}{|l|}{ EN } & \multicolumn{2}{|c|}{ LDUQ } & \multicolumn{2}{|l|}{ IV } \\
\hline & Frecuencia & $\%$ & Frecuencia & $\%$ & Frecuencia & $\%$ & Frecuencia & $\%$ \\
\hline $\begin{array}{c}\text { Hemorragia } \\
\text { digestiva, } \\
\text { Perforación } \\
\text { gástrica, } \\
\text { riesgo de } \\
\text { infarto de } \\
\text { miocardio, } \\
\text { coágulos en } \\
\text { las piernas, } \\
\text { elevan la } \\
\text { presión } \\
\text { arterial, } \\
\text { posible } \\
\text { insuficiencia } \\
\text { renal. }\end{array}$ & 10 & 33 & 10 & 33 & 12 & 40 & 14 & 47 \\
\hline $\begin{array}{l}\text { Daño hepático } \\
\text { leve, } \\
\text { posibilidad de } \\
\text { insuficiencia } \\
\text { renal, vómito, } \\
\text { tensión } \\
\text { muscular, } \\
\text { mareos. }\end{array}$ & 17 & 57 & 19 & 63 & 15 & 50 & 15 & 50 \\
\hline $\begin{array}{l}\text { Hemorragia } \\
\text { pulmonar, } \\
\text { perforación } \\
\text { gástrica, } \\
\text { vómito, } \\
\text { posibilidad de } \\
\text { ceguera, } \\
\text { infarto al } \\
\text { miocardio. }\end{array}$ & 3 & 10 & 1 & 3 & 3 & 10 & 1 & 3 \\
\hline Total & 30 & $100 \%$ & 30 & $100 \%$ & 30 & $100 \%$ & 30 & $100 \%$ \\
\hline
\end{tabular}

Así mismo, a través de una nueva pregunta, se determinó que la mayoría de futbolistas sí están conscientes de los daños que causa a su organismo la automedicación con fármacos que traten el dolor, fiebre e inflamación.
Finalmente, y en función de las respuestas de los encuestados respecto al conocimiento del concepto de AINE, cuestión planteada en una pregunta diferente; se presenta la Tabla 5. 
Tabla 5. Conocimiento sobre conceptualización de los AINE.

\begin{tabular}{cccccccccc}
\hline \multirow{2}{*}{ Opciones } & \multicolumn{2}{c}{ UC } & \multicolumn{2}{c}{ EN } & \multicolumn{3}{c}{ LDUQ } & \multicolumn{2}{c}{ IV } \\
& Frecuencia & $\%$ & Frecuencia & $\%$ & Frecuencia & $\%$ & Frecuencia & $\%$ \\
\hline Sí & 2 & 7 & 4 & 13 & 8 & 27 & 15 & 50 \\
No & 28 & 93 & 26 & 87 & 22 & 73 & 15 & 50 \\
\hline Total & $\mathbf{3 0}$ & $\mathbf{1 0 0 \%}$ & $\mathbf{3 0}$ & $\mathbf{1 0 0 \%}$ & $\mathbf{3 0}$ & $\mathbf{1 0 0 \%}$ & $\mathbf{3 0}$ & $\mathbf{1 0 0 \%}$ \\
\hline
\end{tabular}

\section{Caracterización de la población}

Tras el análisis de la información recopilada, se evidenció que la edad de los futbolistas profesionales de las plantillas de primera división de cada equipo en estudio, oscila entre los 15 y 34 años. Como es de conocimiento general, muchos de los niños; sobre todo en América Latina, sueñan con ser futbolistas profesionales y alcanzar lo más alto en este deporte. Pero, los sacrificios que deben hacer para alcanzar ello, son innumerables. Parte de esto es dejar de lado el desarrollo "normal" de ciertas etapas tales como: niñez, adolescencia y hasta adultez. Por ello, el talento es importante para llegar a la élite, pero el trabajo y el sacrificio para ser futbolista profesional deben siempre ser las premisas para cualquiera que quiera dedicar su vida al deporte.

\section{Conocimiento del grupo farmacológico y principios activos que alivian dolor, fiebre e inflamación}

En lo referente al conocimiento del grupo farmacológico que contrarresta síntomas de dolor, fiebre e inflamación; se determinó que existe gran confusión y hasta cierto punto ignorancia en relación a la identificación de medicamentos; pues tres de los cuatro equipos consideran que son los antibióticos aquellos fármacos que alivian cuadros de dolor, fiebre e inflamación. Cuando en realidad y tal como lo expresa PortalFarma (7) los antibióticos son un tipo de medicamentos que se utilizan para el tratamiento y prevención de enfermedades producidas por bacterias, pues actúan frente a ellas destruyéndolas o bien impidiendo su reproducción.

Así mismo debe rescatarse, que el único equipo que mostró conocimiento sobre usos terapéuticos de los AINE fue Independiente del Valle, razón que apoya a lo escrito en Diario El Universo (8): “...pero en Independiente del Valle no solo se juega fútbol, dice Daniel Reyes, quien, por las tardes, desde las 16:00, acude al colegio con sus compañeros. Una de las visiones del club del Independiente del Valle es formar personas íntegras por medio del deporte, la educación y la disciplina”.

En lo que concierne a la identificación específica de principios activos que tratan dolor, fiebre e inflamación, se observó que existe una gran preferencia por Ibuprofeno, Paracetamol y Diclofenaco; aduciendo a que son los AINE más populares dentro de la población. Estas percepciones no están erradas, pues según el portal Arthritis Foundation (9), los AINE son medicamentos que se usan para tratar tanto el dolor como la inflamación. Y, tomando casos específicos como el del Ibuprofeno cuya acción se centra en inhibir la síntesis de prostaglandinas, que son sustancias responsables de la 
respuesta inflamatoria y del dolor. Por ello, el ibuprofeno como principio activo, se utiliza principalmente como antiinflamatorio y analgésico. Así mismo, el Paracetamol actúa calmando el dolor y la inflamación, tratando lesiones o trastornos que no se acompañen de inflamación, como dolor de cabeza y los síntomas ocasionados por gripe o la fiebre (10).

\section{Frecuencia de consumo de fármacos que alivian dolor, fiebre e inflamación y modo de actuación frente a la obtención de los mismos}

Se evidenció que todos los jugadores coinciden en que hacen uso de los medicamentos en mención nada más cuando se presenta una lesión, argumentando su respuesta en que dejan todo en la cancha o los entrenamientos son muy pesados; por lo demás no los consideran necesarios.

Estos datos se apoyan a lo siguiente: "Evidentemente, el ibuprofeno, la aspirina o el paracetamol pueden usarse, bajo prescripción médica, cuando se sufre una lesión. Sin embargo, hacerlo con anterioridad para evitar una posible lesión o prevenir dolores musculares y así mejorar el rendimiento, las sensaciones o la calificación, conlleva una serie de consecuencias que deben conocerse" (11).

Respecto al cuestionamiento que pretendió recabar información sobre el actuar de los futbolistas frente a una dolencia, se comprobó que casi en su totalidad toman únicamente los medicamentos que indica el doctor del club; pues reconocen que es el experto en el tema. Ahora, estas respuestas se contraponen a un estudio previo realizado en años anteriores por la autora de la actual investigación, en el Club Sociedad Deportivo Aucas, donde, respondiendo a la misma pregunta se evidenció lo siguiente: “... la práctica de auto medicación que mantienen los jugadores. El $74 \%$ de los sujetos en estudio aseveran acudir a establecimientos farmacéuticos para adquirir medicamentos adicionales a los que reciben en el departamento médico del club. Estos datos demuestran que la falta de conocimiento unida a la desesperación de acelerar la recuperación tras sufrir alguna lesión son una constante dentro de la plantilla principal del C.S.D. "Aucas"'” (12).

Entonces, se puede mencionar que, en un lapso de 5 años, el aspecto educativo y sanitario ha cambiado en los jugadores de fútbol; pues en función de las respuestas ya no acuden a un establecimiento farmacéutico con la asiduidad de antes, disminuyendo así la práctica de auto medicación.

\section{Vías de administración preferidas y conocimiento de efectos adversos de los AINE}

Tras el análisis estadístico correspondiente, la vía oral es la que prefiere un gran porcentaje de futbolistas para tomar los medicamentos que alivian el dolor, fiebre e inflamación. Esta percepción se apoya en siguiente: "la vía oral constituye la vía más utilizada de administración de fármacos, aunque se debe tomar en cuenta los factores que pueden influir en su biodisponibilidad (velocidad y magnitud). Así mismo es importante destacar que, en porcentajes variados, la segunda opción en todos los equipos es la vía intramuscular; pues la administración es más adecuada (muy rápida en soluciones acuosas y más lenta en las oleosas), pero se debe tener en cuenta que pueden lesionarse vasos y nervios; así como aparecer parálisis del nervio ciático, hematomas, endurecimientos, infecciones y shock anafiláctico" (13). 
El conocimiento sobre los efectos adversos que ocasionan los AINE en el organismo, es una de las interrogantes de más peso en esta investigación; pues tras el análisis de datos se constató que menos de la mitad del total de futbolistas reconocen los efectos adversos de los fármacos en estudio, lo que genera preocupación; pues forma parte de una educación sanitaria esencial en este tipo de profesión. Sin duda, la automedicación con AINE (antiinflamatorios no esteroideos) en el deporte está más extendida de lo que nos podamos llegar a imaginar. La práctica no es inocua ni ninguna broma: diversos estudios científicos avalan que el consumo de antiinflamatorios analgésicos de forma preventiva antes o durante el ejercicio no sólo no aporta beneficios, sino que puede provocar importantes perjuicios para el organismo, fundamentalmente, intestinales (14).

\section{Efecto perjudicial en la adaptación muscular al ejercicio}

Indudablemente el dolor muscular tiene efectos negativos sobre el desempeño de los deportistas y de las personas que practican ejercicio ocasionalmente, por lo que, para contrarrestarlo y mejorar las marcas y tiempos, se hace un uso preventivo con los AINE. Como mencionan ambos investigadores, cuando un deportista es sometido a un estímulo intenso lo natural es que el cuerpo reaccione con la aparición de dolor o aumento de volumen, pero también se generará como respuesta un proceso de adaptación que permitirá asumir ese mismo estímulo con posterioridad. El dolor muscular forma parte de la adaptación del músculo al entrenamiento. El consumo de antiinflamatorios puede afectar al desarrollo de esa adaptación (15).

\section{Riesgo de sufrir una lesión aún más grave}

Debe mencionarse que antes del ejercicio la toma de ibuprofeno -es el más habitual- no es necesaria y debe evitarse porque contribuye a elevar la presión arterial más de lo que lo hace el ejercicio en sí mismo; esto, junto con la deshidratación propia del ejercicio prolongado, puede facilitar que aparezca insuficiencia renal aguda. Otra consecuencia del ejercicio físico intenso y prolongado es la rabdiomiolisis (microrroturas musculares) que conlleva al uso de AINE para mitigar el dolor (16).

\section{Problemas gastrointestinales}

Fisiológicamente tiene sentido que el ejercicio afecte a los intestinos como lo hace, porque, durante el ejercicio prolongado, la digestión se convierte en un lujo. Así que la sangre que normalmente fluye hacia el intestino delgado se desvía hacia los músculos en pleno esfuerzo. La falta de sangre, hace que algunas células del recubrimiento de los intestinos sufran daños y empiecen a flaquear; es así que el consumo de ibuprofeno puede generar daños gastrointestinales. Determinando que está claro que el consumo de ibuprofeno por deportistas no es inofensivo y debe ser rotundamente desaconsejado (17).

\section{Ibuprofeno y deportes: No solo no ayuda: es perjudicial}

En algunos estudios se comparó el dolor y la inflamación que sufrían los corredores que consumían ibuprofeno durante la prueba y los que no lo hacían, y los resultados fueron unívocos. El ibuprofeno no afectó a la percepción del dolor muscular y, además, los análisis de sangre determinaron que los consumidores de este medicamento registraron mayores niveles de inflamación que aquellos que no lo tomaron. Algunos 
incluso presentaron signos de un leve fallo renal. No existen razones científicas para usar ibuprofeno antes del ejercicio y sí muchas razones para evitarlo (18).

\section{Conocimiento del concepto de AINE y sus efectos a largo plazo en el organismo}

En lo que respecta al criterio de conciencia respecto al daño que varios futbolistas causan a sus organismos por el consumo desmedido de AINE, se denotó que aun cuando presentan ciertos vacíos conceptuales; la idea está clara y saben el perjuicio que se generan así mismos.

En última instancia se observó que, lastimosamente más de la mitad de los futbolistas de cada equipo desconoce la conceptualización de los AINE, aun cuando los toman con frecuencia debido a su profesión. He aquí la importancia de una educación sanitaria adecuada al interior de cada equipo, la explicación - educación farmacéutica respectiva para cada medicamento que se les prescribe, efectos adversos y contraindicaciones; pues en la actualidad, se han producido grandes cambios sociales y tecnológicos que han conducido a la variación de la necesidad social en relación al uso de los medicamentos, lo que nos introduce gradualmente en un tercer periodo, diferente, de la historia de la farmacia, denominado Atención Farmacéutica, representando, esta última, uno de los más importantes marcos epistemológicos de la profesión a lo largo de su historia, al trasladar el foco de la actividad farmacéutica del medicamento hacia el paciente (19).

\section{CONCLUSIONES}

$\mathrm{P}$ ara finalizar, en relación al cumplimiento de los objetivos planteados en este estudio asociados con la identificación del nivel de conocimiento sobre AINE que presentan los futbolistas profesionales de las plantillas de primera división, a través del análisis y realidad de cada equipo y, reconocimiento de los diferentes tipos de AINE utilizados con mayor frecuencia por los futbolistas, vías de administración y las afecciones que pueden provocar en el organismo; se puede mencionar lo siguiente:

Los principales AINE que consumen los futbolistas de los equipos investigados son: ibuprofeno, paracetamol y diclofenaco; mismos que son administrados en su gran mayoría por vía oral.

Así mismo, se constató que existían falencias referentes al conocimiento de medicamentos (AINE) que consumían los futbolistas. Además, se notó que debido a la premura con la que requieren volver a las canchas, éstos profesionales del deporte, suelen confiar en personas que no se encuentran capacitados en medicina o en temas farmacológicos.

Por otra parte, se hizo énfasis en la necesidad de que cada club permita que sus jugadores accedan a este tipo de información, pues, la mayoría (por no decir todos) son cabezas de hogar, y si afectan su salud a través de la automedicación, lastimosamente podrán desarrollar patologías que obligarán a terminar su carrera profesional antes de lo establecido. Pues, si se observa más a fondo el mundo futbolístico se encuentra más que un ejército de jugadores dispuestos a todo con tal de ganar; hallamos a seres humanos que por el desconocimiento atentan contra sus propias vidas, poniendo en riesgo no solo su futuro; sino el de sus familias.

Además, la formación que reciben al interior de cada club es de suma importancia para la vida de los futbolistas, por ello, las divisiones de reserva no deben estar desatendidas; sino incluidas en temas que 
sirven para su crecimiento como excelentes deportistas y como seres humanos.

Finalmente, debido a la novedad de la investigación, los futbolistas de los clubes analizados; en especial los extranjeros, supieron exponer que es de vital importancia que esta información se imparta al interior de todos los equipos del país; ya que por el desconocimiento total que existe en el entorno acerca de los efectos adversos que presentan los AINE muchos de los futbolistas del Ecuador ponen en riesgo sus vidas.

\section{REFERENCIAS BIBLIOGRÁFICAS}

1. Tapia A, Hernández A. Fútbol: conceptos e investigación. EFDeportes.com. 2010.

2. EA SPORTS [Internet]. Sports United. 2016 [citado 13 enero 2020]. Disponible en: http://www.easports.com.eng

3. Federación Internacional de Fútbol FIFA [Internet]. FIFA. 2012 [citado 19 febrero 2020]. Disponible en: https://es.fifa.com/

4. Mucha farmacia en el fútbol. El País [Internet]. 2008 [citado 15 febrero 2020]; Disponible en https://elpais.com/diario/2008/11/0 1/deportes/1225494001_850215.html

5. Medicine and Sports. Medic Sports. 2016;24 a la 26.

6. Federación Ecuatoriana de Fútbol [Internet]. Federación Ecuatoriana de Fútbol (FEF). 2018 [citado 21 febrero 2020]. Disponible en: http://www.ecuafutbol.org/web/

7. Antibióticos [Internet]. PortalFarma.com. 2017 [citado 21 febrero 2020]. Disponible en: https://www.portalfarma.com/Ciuda danos/saludpublica/consejosdesalud/ Paginas/2501antibioticos.aspx

8. Jugando y estudiando, así se forman los chicos del Independiente del Valle. El Universo [Internet]. 2016 [citado 1 marzo 2020]; Disponible en: https://www.eluniverso.com/deporte s/2016/07/16/nota/5693040/jugan do-estudiando-asi-se-forman-chicosindependiente-valle

9. Antiinflamatorios no Esteroideo [Internet]. Arthritis Foundation. 2016 [citado 1 marzo 2020]. Disponible en: http://espanol.arthritis.org/espanol/t ratamientos/medicamentos/medicam entos-porenfermedad/medicamentos-aine/

10. Blog de Kern Pharma: Ibuprofeno vs. Paracetamol [Internet]. Kern Pharma. 2016 [citado 2 marzo 2020]. Disponible en: https://www.kernpharma.com/es/bl og/ibuprofeno-versus-paracetamolconoces-las-diferencias

11. MEDICINA DEL DEPORTE: Antes de entrenar, ¿un ibuprofeno? [Internet]. Saludeporte by Grupo Belman. 2018 [citado 2 marzo 2020]. Disponible en: https://www.saludmasdeporte.com/a ntes-de-entrenar-un-ibuprofeno/

12. Ríos MB. Uso de los AINE en el deporte profesional. Elaboración de un manual y capacitación sobre el uso correcto de los AINE en los futbolistas profesionales del Club Sociedad Deportivo "Aucas". DMQ 2014 [Tecnológico]. Instituto Tecnológico Superior Cordillera; 2014.

13. Ferrandis Tebar V. Formas Farmacéuticas y Vías de Administración. Barcelona: Colegio Profesional Fisioterapeutas de Castilla y León; 2018. 
14. Gavira C. Antes de entrenar un ibuprofeno [Internet]. Salud más Deporte. 2018 [citado 4 marzo 2020]. Disponible en: https://www.saludmasdeporte.com/a ntes-de-entrenar-un-ibuprofeno/

15. Candía - Luján R, De Paz Hernández JA. ¿Son efectivos los antiinflamatorios no esteroideos en el tratamiento del dolor muscular tardío? México D.F.: Medicina y Ciencias de la salud; 2014.

16. Niño Bernal $S$. Todo seguro médico [Internet]. Clínica Beiman. 2018 [citado 5 marzo 2020]. Disponible en: https://www.todosegurosmedicos.co $\mathrm{m} /$ cuadro-medico/detalle/24801caser/
17. Van Wijck K, Van Loon L, Peters W, Buurman W, Dejong C. Exerciseinduced splanchnic hypoperfusion results in gut dysfunction in healthy men. PLoS One. 2011;6 y 7.

18. Nieman D, Henson D, Dumke C, Oley K, McAnulty S, Davis J. Ibuprofen use, endotoxemia, inflammation, and plasma cytokines during ultramarathon competition. Brain, behavior, and immunity. 2006;578 a la 584.

19. Sánchez A. Educación farmacéutica: hacia la enseñanza y el aprendizaje focalizado en el paciente. OFIL. $2017 ; 27$ 